\title{
Purity Predictive Model-based Control of Oxygen Vacuum Swing Adsorption Process
}

\author{
J. Macron, O. Roy, J. Pierquin, P. Rouchon
}

\begin{abstract}
The paper deals with both dynamic modeling and control of the oxygen vacuum swing adsorption (VSA) process. This process is well known for its batch-like operation and inherent non-linearities. The main objectives are to establish a robust dynamic model and then to demonstrate the practical application of a purity predictive model-based control to this industrial system. Experimental results point out the efficiency of the proposed strategy.
\end{abstract}

\section{INTRODUCTION}

$\mathrm{V}$ SA is a method for the production of relatively lowpurity oxygen $(\sim 90 \%)$ which is now a mature and widely used technology in many chemical engineering processes such as glass, iron and aluminum smelting [1],[2]. However, unlike other unit operations, VSA processes remain difficult to understand and, coupled by the lack of theoretical developments in batch process control, are still difficult to control. Furthermore, the focus on previous research efforts in VSA processes has primarily been the determination of cyclic steady-state conditions and has often neglected dynamic aspects. Some attempts of dynamic control do exist but remaining purely theoretical and/or with limited practical applications (such as PID decentralized controls on pilot plant) [3], [4], [5].

The first part of this study deals with the dynamic modeling of an industrial VSA unit. A simplified dynamic model is developed and then validated on a plant delivering oxygen for a glass maker. It is based on a reduced-order representation of VSA physical phenomenon and differs from many previous studies which were based on empirical modeling techniques or comprehensive adsorption simulators limited to single-bed configuration. The responses (oxygen purity, pressure, and flow) of an industrial VSA unit to perturbations in product delivery and weather conditions are validated in comparison with model's outputs. From this dynamic model, a predictive control of the oxygen purity variable (arguably the variable of most importance on this batch system) is designed. The

Manuscript received January 31, 2010.

J. Macron is with the Air Liquide Research Center, Les Loges en Josas, France (phone: +33139076572; e-mail: jonathan.macron@ airliquide.com).

O. Roy is the Air Liquide Standard Plant Engineering, Vitry, France (email: olivier.roy@airliquide.com).

J. Pierquin was with the Air Liquide Research Center. He is now with Advencis, Molsheim, France (e-mail: joseph.pierquin@advencis.com).

P. Rouchon is with the Ecole Nationale Supérieure des Mines de Paris, Centre Automatique et Systèmes, Paris, France (e-mail: pierre.rouchon@ensmp.fr). performance of this control for load rejection, set point changes and weather conditions variations is evaluated. At last, the practical implementation of the advanced process control is discussed.

\section{2. DYNAMIC MODELING OF VSA PROCESS}

\section{A. VSA subsystems \& cycle description}

An industrial VSA unit (depicted in Fig. 1) is usually equipped with:

- Zone 1: two adsorbers supplied by an air blower and connected to a vacuum pump. Two layers of adsorbing components are used in each adsorber: alumina, to catch humidity of air stream, and zeolithes, to adsorb nitrogen.

- Zone 2: a buffer able to store a relatively large volume of oxygen (in comparison with adsorbers) and then to limit pressure variations.

- Zone 3: a compressor to control pressure and/or flow rate delivered to the final customer through a recycling valve.

- Zone 4: a cryogenic vessel filled with Liquid Oxygen (LOX), used when customer's demand exceeds VSA own capability.

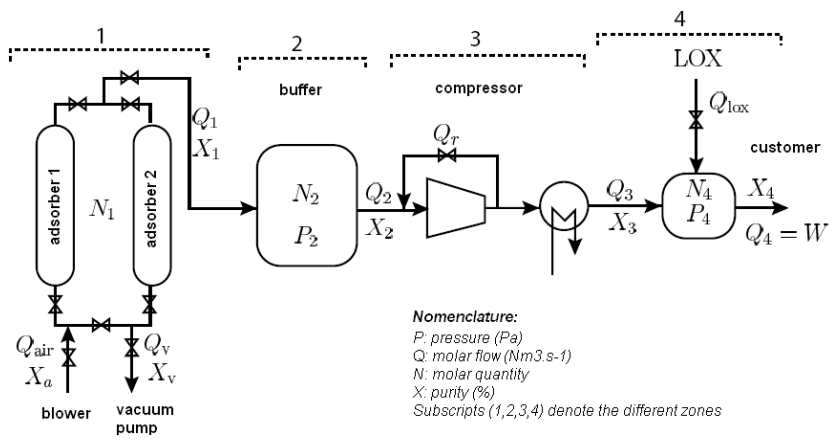

Fig. 1. Subsystems of a VSA industrial unit

An operation cycle consists of 5 main steps (for type-M Air Liquide VSA Unit). Both adsorbers are run in a phase-shifted manner in order to obtain a quasi-continuous production (Fig. 2). Typical pressure profiles in both adsorbers (Zone 1 - Adsorbers $1 \& 2$ ) and buffer (Zone 2 - Buffer) are given in Table 1. A detailed description of the various steps within the cycle, step times, flow direction and control valves for a typical VSA can be found in [1]. 


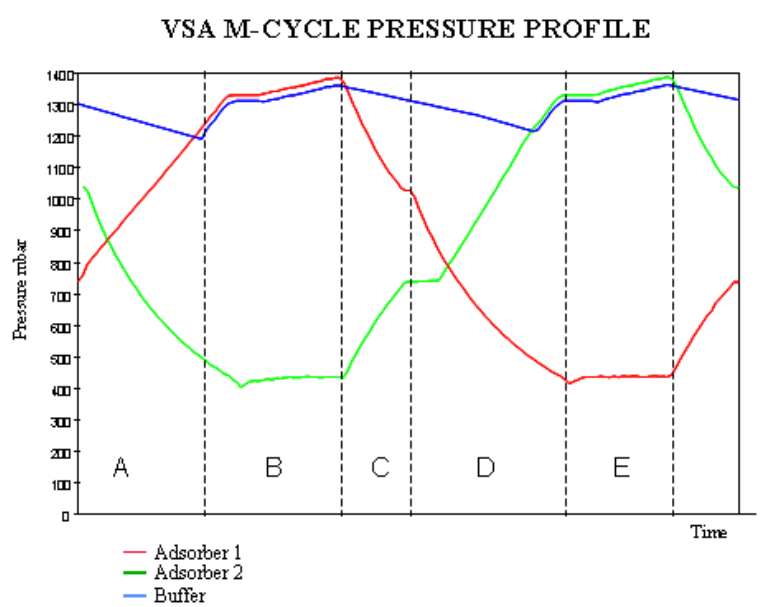

Fig. 2. Pressure cycle in adsorbers \& buffer

TABLE I

5-STEPS 02 VSA CYCLE

\begin{tabular}{cl}
\hline \hline Phase & \multicolumn{1}{c}{ Sequence } \\
\hline A & Air admittance \& pressurization of adsorber 1 \\
B & O2 production by adsorber 1 \\
C & O2 production by adsorber 1 (while purging adsorber 2 \\
& to regenerate its adsorbers) \\
D & Depressurization of adsorber 1 \\
E & Purging of adsorber 1
\end{tabular}

\section{B. VSA Purity: Average Dynamic Model}

The model presented is this section is mainly based on the application of mass balance equations to the different subsystems (zones) and on the related transit times. The control strategy is presented in section 3: it aims at controlling the purity $X$ (or $X_{3}$ ) at the outlet of the VSA by adjusting the flow rate $Q_{\text {ref }}$ delivered to the customers.

Therefore, for zone 1 (adsorbers), bass balance equation is given by:

$\frac{d}{d t}\left(N_{1} X_{1}\right)=Q_{\text {air }} X_{\text {air }}-Q_{1} X_{1}-Q_{v} X_{v}$

Where $N_{I} X_{I}$ is the total number of mole in zone 1 which is equivalent to consider an average model over an entire cycle. By considering zone 1 perfectly homogeneous, equation (1) becomes:

$N_{1} \frac{d}{d t} X_{1}=Q_{a i r}\left(X_{a i r}-X_{1}\right)-Q_{v}\left(X_{v}-X_{1}\right)$

Which can be simplified as $X_{v}<<X_{I}$ :

$N_{1} \frac{d}{d t} X_{1}=Q_{a i r} X_{a i r}-\left(Q_{a i r}-Q_{v}\right) X_{1}$

A last simplification, based on the following relation, gives:

$\tau_{1} \frac{d}{d t} X_{1}=X_{\text {air }}-\frac{Q_{1}}{Q_{\text {air }}} X_{1}$

Where $\tau_{l}$ is the so-called transit time in adsorbers equal to:
$\tau_{1}=\frac{N_{1}}{Q_{\text {air }}}$

As the control objective is to control the purity delivered to the customer it should be noted that the time constant related to $X_{I}$ is:

$\frac{\tau_{1}}{r}=\frac{\tau_{1} X_{\text {air }}}{X_{1}}=\frac{N_{1} X_{\text {air }}}{Q_{\text {air }} X_{1}}=95 \mathrm{~s}$

Where $r$ is the ratio between the oxygen in the air $(\sim 20 \%)$ and the oxygen delivered to the customer ( $\sim 90 \%)$. It should be noted that $\tau_{1}$ depends on $Q_{\text {air }}$ (which can be considered as constant at $4275 \mathrm{Nm}^{3} / \mathrm{h}$ ) and $Q_{1}$ which corresponds to customer's flow rate in steady state $\left(Q_{1}=Q_{2}=Q_{3}=Q_{4}=950 \mathrm{Nm}^{3} / \mathrm{h}\right)$.

By supposing that the buffer composition in zone 2 is homogeneous, a similar behavior can be described by the following equation:

$\frac{d}{d t}\left(N_{2} X_{2}\right)=Q_{1} X_{1}-Q_{2} X_{2}$

From which can be deduced:

$\tau_{2} \frac{d}{d t} X_{2}=X_{1}-X_{2}$

Where $\tau_{2}$ is the transit time in the buffer given by:

$\tau_{2}=\frac{N_{2}}{Q_{1}}=295 \mathrm{~s}$

Zone 3 (compressor \& re-circulating valve) does not affect the product purity \& flow. Then:

$Q_{2}=Q_{3}=Q \quad, \quad X_{2}=X_{3}=X$

The only dynamic phenomenon in this zone is induced by the flow control loop which can be considered as a first order system, with a constant time $\tau_{Q}$ (equal to $40 \mathrm{~s}$ ):

$\tau_{Q} \frac{d}{d t} Q=Q_{\text {ref }}-Q$

By combining and linearizing equations (4), (9) and (12), a third order linear model $F(s)$ is deduced to link $Q_{\text {ref }}$ and $X$ :

$X=F(s) Q$

\section{VSA Purity: Average Dynamic Model}

The model described above has been compared to data from on an industrial unit delivering oxygen for a glass maker in the Paris area.

The variations of purity of the oxygen delivered to the customer have been observed in response to demand (flow) steps $\left(Q_{\text {ref }}\right.$ - see Fig. 3). It is observed that the oxygen purity has an inverse response as described in equation (4). The global experimental response time is approximately $500 \mathrm{~s}$, while the dominant constant time $\left(\tau_{2}\right)$ of the model previously defined was closer to $300 \mathrm{~s}$. 

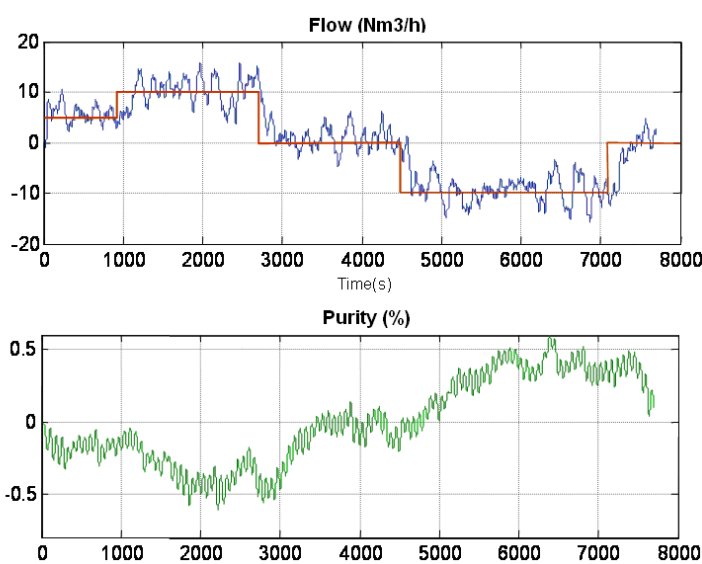

Fig. 3. VSA Oxygen Purity $(X)$ response to flow steps $\left(Q_{r e f}\right)$. Only variations are represented. Nominal Flow @ $950 \mathrm{Nm}^{3} / \mathrm{h}-$ Nominal Purity@ 90\%

Thus, the oxygen purity exhibited a measurable delayed reaction to the change (dead time $\sim 100$ s) and a significantly slower return to cyclic steady state.

It should be recalled that the oxygen product flows into a large product tank and that the oxygen purity reported here (and of interest for the customer) was measured downstream of the buffer. The oxygen response was therefore strongly influenced by the mixing patterns in the product tank. If perfect mixing occurred, the response time would be longer and clearly of a different type (exponential decay to the steadystate value). It is therefore suggested that the primary reason for the time delay in oxygen response is the existence of a composition gradient in the buffer. Equation (13) is then modified to take this delay into account:

$$
X=F(s) e^{-T d s} Q
$$

Model given by equation (13) is then validated on experimental data as shown in Fig. 4.

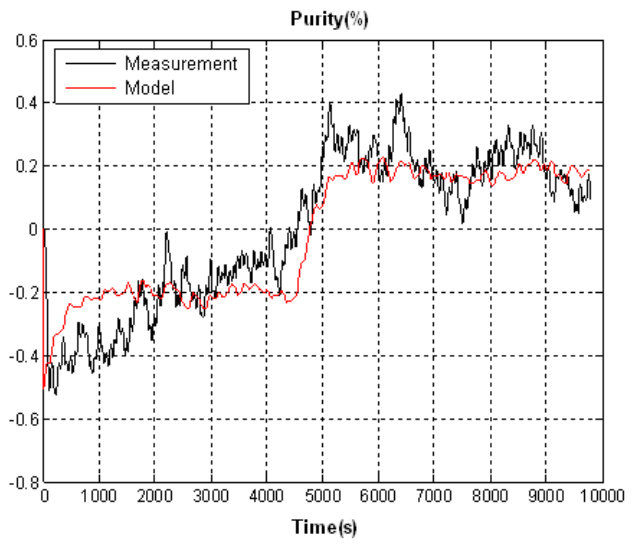

Fig. 4. Experimental validation of VSA oxygen purity model

\section{PREDICTIVE PURITY CONTROL}

\section{A. Economical Interest of Oxygen Purity control}

Predictive Functional Control (PFC), belonging to the family of predictive control techniques, has been demonstrated as a powerful algorithm for controlling process plants. It is here implemented to control the VSA Oxygen purity which is arguably the variable of most importance on the plant, especially from the perspective of a customer and consequently demands tight control tolerances (typically $\pm 0.5 \%$ of set point).

From a producer standpoint, obtaining the required purity has a strong economical interest. Indeed, failure to achieve product purity required by the customer can induce:

- Product gas venting when purity target is not reached (VSA shutdown). In that case, the customer is supplied by the liquid oxygen backup.

- Overconsumption of LOX in case of purity below its contractual value. It has been seen that flow and purity have inverse responses: any excess of customer's demand dramatically reduces the purity provided by the VSA unit which has to be compensated by LOX injection.

The most common disturbance in oxygen purity control is changes in ambient temperature, which are due to both diurnal and seasonal fluctuations. This type of disturbance affects both the inlet stream temperature and the amount of heat lost or gained by the adsorbent beds and can alter the adsorptive capacity of the zeolite sieve. It is through this mechanism that the performance of the process can vary.

\section{B. Oxygen Predictive Control}

A single loop PFC operates on the following principles [6], [7]:

- internal model,

- reference trajectory,

- auto-compensation, and

- calculation of the manipulated variable.

The PFC implemented is based on an internal model under a cascaded form by observing the fact that any $m$-th order system can be decomposed into a set of first order blocks [7]. Then, the average dynamic purity model is represented in Fig 5.

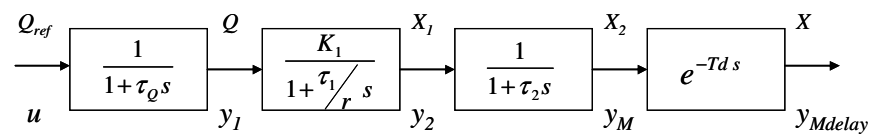

Fig. 5. Internal model in a cascaded form 
By using the classical input/output formalism $(u / y)$, the discrete time formulation of the model zero-order hold equivalent is given by [7]:

$G_{M}(z)=\prod_{i=1}^{M} \frac{K_{i}\left(1+\beta_{i} z^{-1}\right)}{1-\alpha_{i} z^{-1}}$

With respect to the instant $\mathrm{k}$, the model output can be predicted as:

$y_{M}(k)=\alpha_{M} y_{M}(k-1)+K_{M} y_{2}(k)+K_{M} \beta_{M} y_{2}(k-1)$

which can be decomposed into free and forced responses by calculating $y_{2}(k)$ and $y_{1}(k)$ in the same way [7].

The reference trajectory used here is based on a second-order response (Butterworth type) to ensure a smooth flow rate variation.

In PFC, the desired response is normally specified as [7]:

$$
R_{r}=\frac{O L R T}{C L R T}
$$

which defines the ratio of the Open Loop Response Time (OLRT, the time to $90 \%$ of the final value) to the Closed Loop Response Time (CLRT). A ratio of 3 is chosen for the VSA process - which is typical of slow processes [7].

The way the delay is taken into account and compensated as well as further details on the formulation of the control law in the case of cascaded first order blocks can be found in [7].

\section{Experimental Results}

The tracking performance of oxygen control loop is given in Fig. 6. It shows a fast response time as expected and a good compensation of the delay.

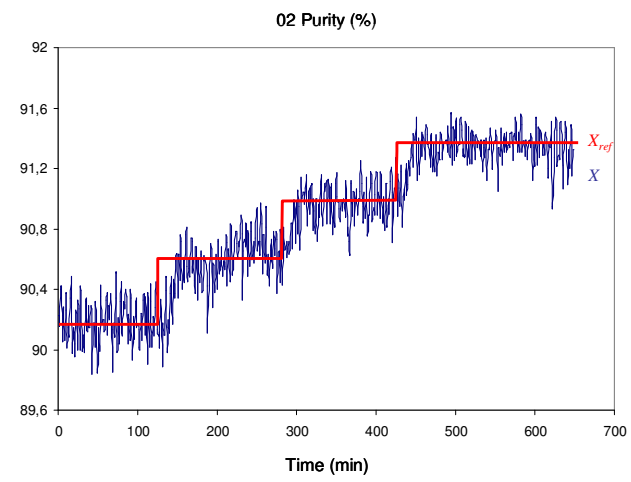

Fig. 6. Purity predictive control: tracking performance
The robustness of predictive control has been demonstrated on a two years testing period. The main process disturbances are both outside pressure and temperature. From this standpoint, the efficiency of the control is confirmed: despite the variations of temperature at the inlet of the system (corresponding to the day \& night cycle), the purity remains stable (Fig. 8) - ranged within a $0.2 \%$ tolerance while the flow is maximized to optimize the unit productivity (Fig. 7).

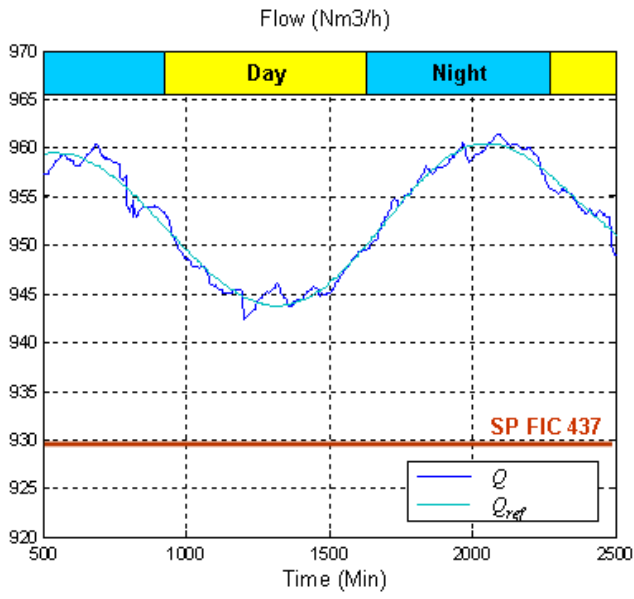

Fig. 7. Day \& Night cycles: flow optimization

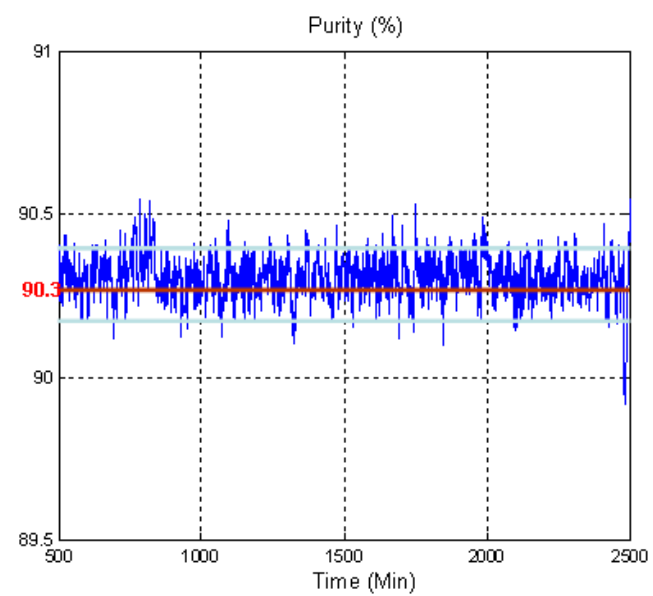

Fig. 8. Day \& Night cycles: Purity control at $\pm 0.2 \%$

To confirm the robustness of the control to a faster disturbance, a variation of the inlet temperature has been applied by quickly increasing the temperature on the heat exchanger (Fig. 9). For this worst case scenario (which could correspond to sudden change of atmospheric conditions - such as a storm for instance), purity remains constant as well (Fig. 10). 


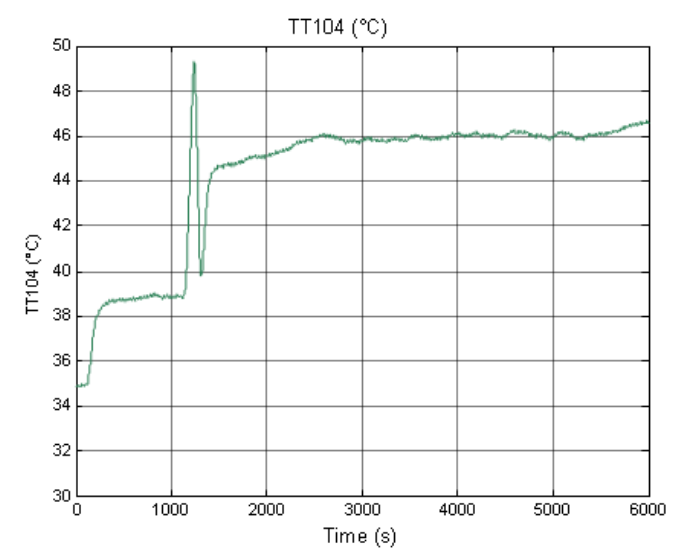

Fig. 9. Inlet Heat Exchanger Temperature

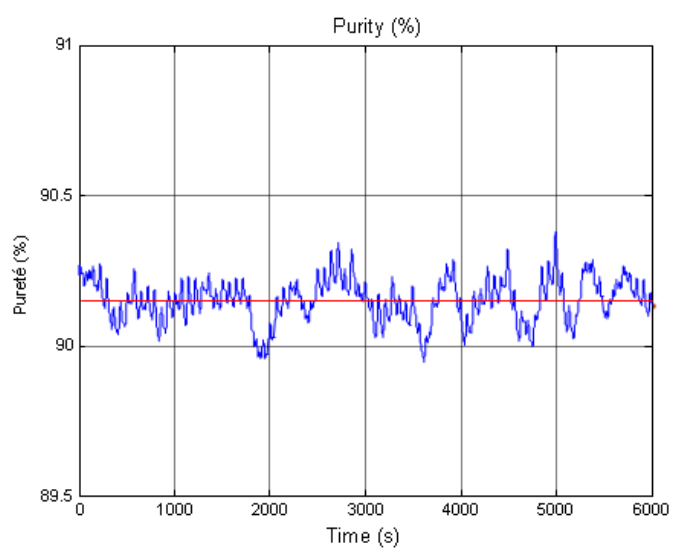

Fig. 10. Oxygen purity during heat exchanger temperature disturbance

At last, the PFC has also led to significant reductions of both energy and liquid oxygen (LOX) consumptions. LOX Savings have been obtained by maximizing the flow rate (Table 2 ), as no manual intervention to adjust purity is needed anymore on both VSAs. This manual adjustment used to be conservative (i.e leading to a higher purity than requested by the customer) to avoid any loss of purity which could have produced a plant shutdown.

TABLE 2

LOX SAVINGS ON 2 VSA UNITS

\begin{tabular}{ccc} 
& \multicolumn{2}{c}{ LOX SAVINGS ON 2 VSA UNITS } \\
\hline \hline Unit & $\begin{array}{c}\text { LOX Savings } \\
\%\end{array}$ & $\begin{array}{c}\text { LOX Savings } \\
\mathrm{Nm}^{3}\end{array}$ \\
\hline VSA 1 & $23,6 \%$ & 116000 \\
VSA 2 & $20,8 \%$ & 102500 \\
Total & $\mathbf{3 9 , 1 \%}$ & $\mathbf{1 9 2} \mathbf{5 0 0}$
\end{tabular}

\section{CONCLUSION}

The paper deals with both dynamic modeling and predictive control of the oxygen vacuum swing adsorption. The performance of this control for both tracking and disturbance rejection (mainly induced by the variations of weather conditions) is demonstrated on an industrial plant over a significant period and led to both productivity increase \& ease of operation. To our knowledge, the results presented in this paper are the first ones in the literature obtained on an industrial unit.

\section{REFERENCES}

[1] D.M. Ruthven, S. Farooq, K.S. Knaebel, "Pressure Swing Adsorption" VCH Publishers, Weinheim, New York, Cambridge, 1994

[2] R.T. Yang, "Gas Separation by Adsorption Processes", Butterworths, Boston, 1987.

[3] Beh,C.C.K., P.A.Webley, "Control of the Oxygen Vacuum Swing Adsorption Process. Part 1 - Single Loop Control", Industrial and Engineering Chemistry Research, 2004.

[4] A.I. Shirley, A. I. LaCava, "PSA System with product turndown and purity control", US Patent n`5.258.056, The BOC Group.

[5] Beh, C.C.K.; Wilson, S.; Webley, P.A.; He, J. "The Control of the Vacuum Swing Adsorption Process for Air Separation", Proceedings of the Second Pacific Basin Conference on Adsorption Science and Technology, 2000, D. Duong (ed), World Scientific, Singapore, 663.

[6] Richalet J, "Pratique de la commande predictive", Hermès, Paris, 1993.

[7] Mohamed Tarek Khadir; John V. Ringwood, "Extension of first order predictive functional controllers to handle higher order internal models", Int. J. Appl. Math. Comput. Sci., 2008, Vol. 18, No. 2, 229-239. 